\title{
Identificación Algebraica de Parámetros Asistida por Observadores GPI para un Helicóptero de Dos Grados de Libertad
}

\author{
Harvey D. Rojas ${ }^{(1,2) \star}$, Herbert E. Rojas ${ }^{(3)}$ y John A. Cortés ${ }^{(4)}$ \\ (1) Universidad Manuela Beltrán, Programa de Ingeniería Electrónica. Avenida circunvalar № 60-00, \\ Bogotá D.C., Colombia, (e-mail: harvey.rojas@docentes.umb.edu.co). \\ (2) Servicio Nacional de Aprendizaje SENA, Centro de electricidad, electrónica y telecomunicaciones, Av. \\ Carrera 30 \# 17B-25-Sur, Bogotá D.C., Colombia, (e-mail: davidrc@misena.edu.co). \\ (3) Universidad Distrital Francisco José de Caldas, Proyecto Curricular de Ingeniería Eléctrica, Grupo \\ GCEM-UD, Cra 7 \# 40-53, piso 5, Bogotá D.C., Colombia. (e-mail: herojasc@udistrital.edu.co). \\ (4) Universidad Nacional de Colombia, Facultad de Ingeniería, Depto. de Ingeniería Eléctrica y Electrónica, \\ Av. Carrera 30 No. 45-03 Edif. 453 Of. 222, Bogotá D.C., Colombia. (e-mail: jacortesr@unal.edu.co).
}

* Autor a quien debe ser dirigida la correspondencia

Recibido Dic. 12, 2017; Aceptado Mar. 6, 2018; Versión final May. 6, 2018, Publicado Oct. 2018

\begin{abstract}
Resumen
Este artículo propone una alternativa para la estimación de parámetros de un helicóptero de dos grados de libertad que opera bajo control de lazo cerrado. Como principal contribución se plantea una variante de la metodología algebraica de identificación, la cual involucra el uso de observadores proporcional integral generalizados (GPI). Estos observadores están encargados de proveer la estimación de las derivadas de las salidas del sistema para su computo directo en los casos donde existen operaciones no lineales de difícil manipulación analítica. Inicialmente, se establece un identificador algebraico para cada ecuación no-lineal del sistema a fin de estimar los parámetros de interés. Posteriormente, para la selección de los parámetros estimados se adopta el criterio de la integral del cuadrado del error y el correspondiente índice de error. Finalmente, se presentan resultados experimentales que permiten validar la estrategia de identificación utilizada y los parámetros estimados con respecto al modelo asumido.
\end{abstract}

Palabras clave: estimación de parámetros; identificación algebraica; identificación en lazo cerrado; observadores GPI; helicóptero 2-DOF

\section{Algebraic Parameter Identification Assisted by GPI Observers for a Helicopter with Two Degrees of Freedom}

\begin{abstract}
This paper proposes an alternative for parameter estimation of a helicopter with two degrees of freedom that operates under closed loop control. The main contribution of this work is a variant of the algebraic identification methodology, which involves the use of generalized proportional integral observers (GPI). These observers are responsible for providing the estimation of the time-derivatives of the system outputs for their direct computation in the cases where there are non-linear operations with difficult analytical manipulation. Initially, in order to estimate the parameters of interest, an algebraic identifier for each non-linear equation of the system is proposed. Subsequently, for the selection of the estimated parameters the criterion of integral squared error and the corresponding error index are adopted. Finally, experimental results that allow validating the identification strategy and the estimated parameters with respect to the model assumed are presented.
\end{abstract}

Keywords: parameter estimation; algebraic identification; closed loop identification; GPI observers; 2-DOF helicopter 


\section{INTRODUCCIÓN}

El conocimiento de los parámetros asociados a los modelos de sistemas industriales, se ha convertido en un reto fundamental en diferentes campos de la ingeniería, tales como el diseño de sistemas de control de alto desempeño (García-Rodríguez et al., 2009), el diagnostico automatizado de equipos (Flores y Asiaín, 2011) y la planeación y ejecución de acciones de mantenimiento (Campuzano-Martínez, 2016), entre otros. Lo anterior, ha incentivado el desarrollo de numerosos métodos de identificación/estimación con aplicación a sistemas lineales y no-lineales, tales como: las técnicas basadas en predicción de error para sistemas lineales (Nelles, 2013), los métodos probabilísticos para el modelamiento de procesos (Kodamana et al., 2018), el uso de aproximaciones lineales para el modelamiento de sistemas no lineales (Schoukens y Tiels, 2017) y la identificación basados en datos de sistemas no lineales complejos (Wang et al., 2016;), entre otros.

Una alternativa desarrollada en los últimos años que ha demostrado una gran rapidez y robustez en la estimación de parámetros y estados, es la basada en métodos algebraicos (Sira-Ramírez et al., 2014a). La metodología de identificación algebraica de parámetros fue introducida por Fliess y Sira-Ramírez (2003), como un método basado en cálculo operacional (para el caso lineal) y consideraciones en el dominio del tiempo. Este método permite obtener los parámetros desconocidos del sistema, mediante un conjunto de ecuaciones lineales variantes en el tiempo, las cuales son independientes de las condiciones iniciales y de las perturbaciones estructuradas (Fliess y Sira-Ramírez, 2003). Adicionalmente, esta técnica no es asintótica en su naturaleza y no requiere un conocimiento estadístico del ruido que afecta las mediciones realizadas sobre el sistema, aspecto que resulta muy atractivo a la hora de la implementación (Fliess et al., 2008).

Los métodos algebraicos han sido probados en múltiples aplicaciones del ámbito académico e industrial. En un primer nivel, estos métodos han mostrado resultados exitosos en el tratamiento de señales sinusoidales para la identificación de modos de vibración en estructuras flexibles (San-Millan y Feliu, 2015), la estimación de contenido armónico en sistemas eléctricos de potencia (Beltrán-Carbajal et al., 2018) y el filtrado de señales afectadas por la presencia de ruido (Morales et al., 2016). En segundo nivel, han sido incorporados en diferentes esquemas de control permitiendo la estimación algebraica de estados y derivadas para aplicaciones de control de movimiento (Menhour et al., 2014), la tolerancia a fallos en sistemas de levitación magnética (Kiltz et al, 2014) y procesos industriales (Martínez-Guerra et al., 2017) y el control de sistemas mecatrónicos basado en el rechazo activo de perturbaciones estimadas algebraicamente (Cortés-Romero et al., 2017). En tercer nivel, los métodos algebraicos han sido utilizados en la estimación de parámetros de sistemas dinámicos lineales y no lineales, demostrando ser útiles en la caracterización de servo-mecanismos (Garrido y Concha, 2013; Miranda-Colorado y Moreno-Valenzuela, 2017), el control de robots (Becedas et al., 2009; Pereira et al., 2013), el control de sistemas mecánicos (García-Rodríguez et al., 2009; Beltrán-Carbajal y Silva-Navarro, 2013), el control de convertidores electrónicos de potencia (Linares et al., 2014; Bougrine et al., 2017) y el control de máquinas eléctricas (Delpoux y Floquet, 2015).

Un aspecto distintivo de la metodología algebraica para la estimación de parámetros, es el uso de manipulaciones algebraicas del modelo matemático del sistema. Esto incluye, en general, la derivación analítica de las ecuaciones, la multiplicación por potencias del tiempo y el uso de integraciones iteradas en el dominio del tiempo (Fliess et al., 2008). Estos procedimientos se realizan a fin de eliminar el efecto de las perturbaciones estructuradas y las condiciones iniciales y obtener ecuaciones libres de derivadas explicitas, donde las expresiones resultantes se pueden organizar en términos de ecuaciones lineales en los parámetros Ahora bien, en los casos donde existen operaciones no-lineales directas entre las variables de estado y las derivadas de las salidas del sistema, la complejidad en el desarrollo analítico aumenta, aspecto que puede llegar a dificultar su implementación práctica (Sira-Ramírez et al., 2014a). Una alternativa para enfrentar dicho problema es el uso de sistemas que proveen la estimación de las señales de difícil manipulación desde la perspectiva algebraica, tales como los observadores proporcional integral generalizados (GPI). Las características y aplicaciones de este tipo de observadores han sido documentadas en múltiples trabajos que abordan problemas como el control de señales periódicas (Cortés-Romero, et al., 2014), control de convertidores electrónicos de potencia (Zurita-Bustamante et al., 2011), sincronización de circuitos caóticos (Luviano-Juarez et al., 2010), control de máquinas eléctricas (Sira-Ramírez et al., 2014b) y aplicaciones de tolerancia a fallos (Cortés-Romero et al., 2013), entre otros.

Teniendo en cuenta lo anterior, este trabajo presenta una variante de la metodología algebraica para la estimación de parámetros e involucra el uso de observadores GPI con dos propósitos: (1) el diseño del controlador que garantice la estabilidad del sistema bajo un enfoque de rechazo activo de perturbaciones; y (2) la estimación de las derivadas de algunas señales, reduciendo así la complejidad en las manipulaciones algebraicas del modelo del sistema y facilitando la implementación final de los identificadores. Para validar el esquema de identificación propuesto se considera, como caso de estudio, el problema de estimación simultánea de un conjunto de ocho parámetros asociados al modelo no-lineal de un helicóptero de dos grados de libertad (2-DOF). Asimismo, se adoptan el criterio de la integral del cuadrado del error y el correspondiente 
índice de error, con el propósito de evaluar el grado de ajuste de los parámetros estimados al modelo del sistema. Finalmente, se presentan los resultados experimentales que validan el esquema de identificación propuesto.

\section{MODELO MATEMÁTICO DEL HELICÓPTERO DE DOS GRADOS DE LIBERTAD}

El helicóptero de dos grados de libertad (2-DOF) es un sistema aerodinámico no-lineal, multivariable y altamente acoplado, cuyo modelo matemático puede ser obtenido a partir de las ecuaciones de movimiento de Lagrange-Euler para un sistema dinámico de dos grados de libertad (Rojas-Cubides et al., 2015). Durante su funcionamiento, el helicóptero puede inclinarse al rededor del eje de $Y$ (Pitch) un ángulo $\theta(t)$ y rotar al rededor del eje Z (Yaw) un ángulo $\psi(t)$. Estas variables representan las salidas del sistema y están disponibles para su medición.

Para la formulación del modelo matemático del helicóptero 2-DOF se han tenido en cuenta las siguientes consideraciones: (a) El vector de coordenadas generalizadas está compuesto por las posiciones y velocidades angulares del sistema $[\theta(t) \psi(t) \dot{\theta}(t) \dot{\psi}(t)]$. (b) Para calcular la energía cinética y potencial, requeridas en la formulación de Lagrange - Euler, se tiene en cuenta que el centro de masa está desplazado con respecto al pivote. Esto hace que la nariz del helicóptero caiga por efecto de la gravedad. (c) Se tomaron en cuenta los efectos de las fricciones viscosas y de Coulomb presentes en los movimientos de inclinación y rotación. (d) Se asumen como constantes las relaciones de voltaje a par producidas por los motores DC de las hélices frontal y trasera. Esto teniendo en cuenta que la dinámica del helicóptero 2-DOF está fuertemente dominada por el componente mecánico y no por el eléctrico. (e) El modelo teórico obtenido es válido para cualquier punto de operación posible en el sistema, es decir, entre $-40^{\circ}$ y $40^{\circ}$ para el ángulo de inclinación $\theta(t)$ y entre $-360^{\circ}$ y $360^{\circ}$ para el ángulo de rotación $\psi(t)$. Estos puntos de operación se establecieron dadas las características constructivas del helicóptero empleado (Quanser Inc., 2012; Rojas-Cubides et al., 2015). A partir de estas consideraciones, las ecuaciones (1) y (2) presentan el modelo matemático utilizado en este artículo.

La ecuación (1) describe el movimiento del helicóptero en el eje de Pitch (inclinación). En esta expresión, el momento de inercia equivalente en el eje de Pitch es $J_{\text {eqp }}$, $M_{\text {heli }}$ es la masa móvil total del helicóptero y las posiciones del centro de masa en los ejes $X$ y $Z$ son identificadas como $l_{m c}$ y $h$, respectivamente. Por su parte, las señales de control $V_{m p}(t)$ y $V_{m y}(t)$ son los voltajes aplicados a las hélices frontal (motor Pitch) y trasera (motor Yaw) y los parámetros $K_{p p}$ y $K_{p y}$ son constantes que relacionan los voltajes aplicados a los motores con los pares generados en el eje de Pitch. El parámetro $B_{p}$ es el coeficiente de fricción viscosa y $F_{c p}$ es una constante debida a la fricción de Coulomb, ambos parámetros evaluados en el eje de Pitch. Finalmente, la primera y segunda derivada del ángulo de inclinación serán expresadas como $\dot{\theta}(t)=d \theta(t) / d t$ y $\ddot{\theta}(t)=$ $d^{2} \theta(t) / d t^{2}$, respectivamente.

$$
\begin{aligned}
& {\left[J_{\text {eqp }}+M_{h e l i}\left(l_{m c}{ }^{2}+h^{2}\right)\right] \ddot{\theta}(t)+M_{h e l i}\left[\frac{1}{2} \sin 2 \theta(t)\left(l_{m c}{ }^{2}-h^{2}\right)-\left(l_{m c} h \cos 2 \theta(t)\right)\right] \dot{\psi}(t)^{2}+} \\
& {\left[M_{h e l i} g\left(l_{m c} \cos \theta(t)+h \sin \theta(t)\right)\right]=K_{p p} V_{m p}(t)+K_{p y} V_{m y}(t)-B_{p} \dot{\theta}(t)-F_{c p} .}
\end{aligned}
$$

Por su parte, la ecuación (2) describe el movimiento en el eje de Yaw, donde $J_{\text {eqy }}$ es el momento de inercia equivalente en el eje de Yaw y los parámetros $K_{y p}$ y $K_{y y}$ son constantes que relacionan el voltaje aplicado a los motores con los pares generados. En esta ecuación, el coeficiente de fricción viscosa en el eje de Yaw es $B_{y}$, mientras $F_{c y}$ es una constante debida a la fricción de Coulomb en dicho eje.

$$
\begin{aligned}
& \left\{J_{e q y}+M_{h e l i}\left[\cos ^{2} \theta(t)\left(l_{m c}^{2}-h^{2}\right)+\left(l_{m c} h \sin 2 \theta(t)\right)+\left(h^{2}\right)\right]\right\} \ddot{\psi}(t)+M_{h e l i}\left[-\sin 2 \theta(t)\left(l_{m c}^{2}-h^{2}\right)+\right. \\
& \left.2\left(l_{m c} h \cos 2 \theta(t)\right)\right] \dot{\psi}(t) \dot{\theta}(t)=K_{y p} V_{m p}(t)+K_{y y} V_{m y}(t)-B_{y} \dot{\psi}(t)-F_{c y} .
\end{aligned}
$$

Los parámetros del helicóptero 2-DOF pueden ser clasificados en tres grupos. El primer grupo está compuesto por los parámetros generales $M_{\text {heli }}, l_{m c}, h, g, J_{\text {eqp }}$ y $J_{\text {eqy }}$. El segundo grupo está conformado por los parámetros $K_{p p}, K_{p y}, B_{p}$ y $F_{c p}$, asociados a la ecuación no-lineal de movimiento en el eje de Pitch (ver ecuación (1)). Finalmente, el tercer grupo está formado por los parámetros asociados a la ecuación de movimiento en eje de Yaw (ver ecuación (2)), es decir, $K_{y p}, K_{y y}, B_{y}$ y $F_{c y}$. Es importante mencionar que los parámetros del segundo y tercer grupo serán objeto de identificación, mientras los parámetros generales del helicóptero 2DOF fueron asumidos de acuerdo a las siguientes consideraciones: (I) la masa móvil total del helicóptero $\left(M_{\text {heli }}\right)$ y los momentos de inercia equivalentes en cada eje $\left(J_{\text {eqp }}\right.$ y $J_{\text {eqy }}$ ) son proporcionados por el fabricante (Quanser Inc., 2012); (ii) el valor de la aceleración de la gravedad ( $g$ ) se considera constante; (iii) el centro de masa del helicóptero no se encuentra en el pivote sino a una distancia $\left(l_{m c}\right)$ a lo largo de la longitud del fuselaje y a una altura $(h)$ por debajo de este. 
Para determinar los parámetros del primer grupo se posicionó una masa conocida en el lado contrario de la nariz del helicóptero de manera que, al variar su ubicación con respecto al pivote, se consiga desplazar el centro de masa del sistema para diferentes ángulos de inclinación medidos. Posteriormente, se desarrolló una ecuación de balance de par alrededor de eje de Pitch (en condiciones de equilibrio y sin voltajes aplicados a los motores), lo cual permite obtener los parámetros asociados a la posición del centro de masa del helicóptero mediante un análisis trigonométrico simple. En la Tabla 1 se presentan los valores de los parámetros generales del helicóptero 2-DOF.

Tabla 1: Parámetros generales del helicóptero 2-DOF

\begin{tabular}{|c|c|c|}
\hline Símbolo & Valor & Unidades \\
\hline$M_{\text {heli }}$ & 1.3872 & $\mathrm{Kg}$ \\
\hline$J_{\text {eqp }}$ & 0.0384 & $\mathrm{Kg} \cdot \mathrm{m}^{2}$ \\
\hline$J_{\text {eqy }}$ & 0.0432 & $\mathrm{Kg} \cdot \mathrm{m}^{2}$ \\
\hline$g$ & 9.8 & $\mathrm{~m} / \mathrm{s}^{2}$ \\
\hline$l_{m c}$ & 0.0122 & $\mathrm{~m}$ \\
\hline$h$ & 0.00714 & $\mathrm{~m}$ \\
\hline
\end{tabular}

\section{CONTROL LINEAL BASADO EN OBSERVADORES GPI DEL HELICÓPTERO 2-DOF}

En esta sección se presenta el diseño del controlador que garantiza la estabilidad del sistema, permitiendo así realizar las pruebas en lazo cerrado. Esto permitirá validar posteriormente la propuesta de identificación.

\section{Representación simplificada y desacoplada del sistema}

Considerando que el helicóptero 2-DOF es un sistema multivariable con dos entradas $\left(V_{m p}(t)\right.$ y $\left.V_{m y}(t)\right)$ y dos salidas $(\theta(t)$ y $\psi(t))$, en este trabajo se propone una representación simplificada y desacoplada de la dinámica descrita por las ecuaciones (1) y (2). Esto se consigue a partir del planteamiento de dos subsistemas diferencialmente planos de una entrada y una salida, tal y como se presentan a continuación (Rojas-Cubides et al., 2015):

$\ddot{\theta}(t)=\kappa_{\theta}(t) u_{\theta}(t)+\xi_{\theta}(t)$,

$\ddot{\psi}(t)=\kappa_{\psi}(t) u_{\psi}(t)+\xi_{\psi}(t)$,

Donde $u_{\theta}(t)$ y $u_{\psi}(t)$ son señales auxiliares de control, calculadas como una combinación lineal de los voltajes de entrada $V_{m p}(t)$ y $V_{m y}(t)$. De esta manera:

$$
\left[\begin{array}{l}
u_{\theta}(t) \\
u_{\psi}(t)
\end{array}\right]=\left[\begin{array}{ll}
K_{p p} & K_{p y} \\
K_{y p} & K_{y y}
\end{array}\right]\left[\begin{array}{l}
V_{m p}(t) \\
V_{m y}(t)
\end{array}\right] .
$$

Esto determina la relación inversa para el cálculo de los voltajes $V_{m p}(t)$ y $V_{m y}(t)$ :

$$
\left[\begin{array}{l}
V_{m p}(t) \\
V_{m y}(t)
\end{array}\right]=\frac{1}{K_{p p} K_{y y}-K_{p y} K_{y p}}\left[\begin{array}{cc}
K_{y y} & -K_{p y} \\
-K_{y p} & K_{p p}
\end{array}\right]\left[\begin{array}{l}
u_{\theta}(t) \\
u_{\psi}(t)
\end{array}\right] .
$$

Por otra parte, $\kappa_{\theta}(t)$ y $\kappa_{\psi}(t)$ (ver ecuaciones (3) y (4)) son funciones que acompañan a las señales auxiliares $u_{\theta}(t)$ y $u_{\psi}(t)$. Estas funciones pueden ser expresadas de la siguiente manera:

$$
\begin{aligned}
& \kappa_{\theta}(t)=\left[J_{\text {eqp }}+M_{\text {heli }}\left(l_{m c}{ }^{2}+h^{2}\right)\right]^{-1}, \\
& \kappa_{\psi}(t)=\left[\left(J_{\text {eqy }}+M_{\text {heli }}\left[\cos ^{2} \theta(t)\left(l_{m c}{ }^{2}-h^{2}\right)+\left(l_{m c} h \sin 2 \theta(t)\right)+\left(h^{2}\right)\right]\right]^{-1} .\right.
\end{aligned}
$$

Finalmente, las señales $\xi_{\theta}(t)$ y $\xi_{\psi}(t)$ son funciones de perturbación generalizadas que reúnen todos elementos asociados a los estados en el modelo y demás términos no incluidos en $\kappa_{\theta}(t)$ y $\kappa_{\psi}(t)$ : 


$$
\begin{gathered}
\xi_{\theta}(t)=\kappa_{\theta}(t)\left\{-M_{\text {heli }}\left[\frac{1}{2} \sin 2 \theta(t)\left(l_{m c}{ }^{2}-h^{2}\right)-\left(l_{m c} h \cos 2 \theta(t)\right)\right] \dot{\psi}^{2}-\right. \\
\left.\left[M_{\text {heli }} g\left(l_{m c} \cos \theta(t)+h \sin \theta(t)\right)\right]-B_{p} \dot{\theta}(t)-F_{e p}\right\}, \\
\xi_{\psi}(t)=\kappa_{\psi}(t)\left[-M_{\text {heli }}\left[-\sin 2 \theta(t)\left(l_{m c}{ }^{2}+h^{2}\right)-2\left(l_{m c} h \cos 2 \theta(t)\right)\right] \dot{\psi}(t) \dot{\theta}(t)-B_{y} \dot{\psi}(t)-F_{c y}\right] .
\end{gathered}
$$

En el análisis anterior, se considera que las funciones que acompañan al control $\kappa_{\theta}(t)$ y $\kappa_{\psi}(t)$ son completamente conocidas ya que dependen de los parámetros generales del sistema presentados en la Tabla 1. De igual manera, como condición necesaria para garantizar que las ecuaciones diferenciales (3) y (4) tengan solución bajo la operación de control en lazo cerrado, se asume que las posibles perturbaciones externas del sistema son uniformemente y absolutamente acotadas. Adicionalmente, para cualquier solución de $\theta(t)$ y $\psi(t)$, obtenidas con las entradas auxiliares $u_{\theta}(t)$ y $u_{\psi}(t)$, las cuales son suaves y uniformemente acotadas, las perturbaciones generalizadas $\xi_{\theta}(t)$ y $\xi_{\psi}(t)$ también son uniformemente y absolutamente acotadas (Guo y Zhao, 2013).A partir de lo anterior, se ha planteado el siguiente problema de control: dadas unas posiciones angulares de referencia $\theta_{r e f}(t)$ y $\psi_{r e f}(t)$ para el helicóptero 2-DOF, se debe proponer una ley de control realimentado de tal forma que se tenga una convergencia, suficientemente cercana, de las salidas medibles, es decir, los posiciones angulares $\theta(t)$ y $\psi(t))$. Esta convergencia debe presentar buenos resultados aún en presencia de incertidumbre en los parámetros, no-linealidades y perturbaciones desconocidas.

\section{Control lineal basado en observador GPI para el eje de Pitch}

Considerando la dinámica simplificada y desacoplada para el lazo de control eje de Pitch mostrada en la ecuación (3), se asume un modelo interno de la perturbación generalizada tal que $d^{3} \xi_{\theta}(t) / d t^{3}=0$. Esto último se plantea conforme a la variación que se espera tenga la perturbación generalizada, la cual incluye los efectos del acople con el otro eje. Con base en lo anterior, y definiendo el error de estimación como $\epsilon_{\theta}(t)=$ $\theta(t)-\hat{\theta}(t)$, se propone el siguiente observador GPI para la estimación de la función de perturbación generalizada y las derivadas de la salida plana en el subsistema desacoplado del eje de Pitch:

$$
\left[\begin{array}{c}
\frac{d \widehat{\theta}(t)}{d t} \\
\frac{d \hat{\theta}(t)}{d t} \\
\frac{d \hat{\xi}_{\theta}(t)}{d t} \\
\frac{d \hat{\xi}_{\theta}(t)}{d t} \\
\frac{d \hat{\xi}_{\theta}(t)}{d t}
\end{array}\right]=\left[\begin{array}{lllll}
0 & 1 & 0 & 0 & 0 \\
0 & 0 & 1 & 0 & 0 \\
0 & 0 & 0 & 1 & 0 \\
0 & 0 & 0 & 0 & 1 \\
0 & 0 & 0 & 0 & 0
\end{array}\right]\left[\begin{array}{c}
\hat{\theta}(t) \\
\hat{\theta}(t) \\
\hat{\xi}_{\theta}(t) \\
\hat{\dot{\xi}}_{\theta}(t) \\
\hat{\tilde{\xi}}_{\theta}(t)
\end{array}\right]+\kappa_{\theta}(t)\left[\begin{array}{l}
0 \\
1 \\
0 \\
0 \\
0
\end{array}\right] u_{\theta}(t)+\left[\begin{array}{l}
l_{\theta 4} \\
l_{\theta 3} \\
l_{\theta 2} \\
l_{\theta 1} \\
l_{\theta 0}
\end{array}\right] \epsilon_{\theta}(t)
$$

Donde el vector de ganancia del observador es $L_{\theta}=\left[\begin{array}{lllll}l_{\theta 4} & l_{\theta 3} & l_{\theta 2} & l_{\theta 1} & l_{\theta 0}\end{array}\right]^{T}$. De esta manera, la dinámica dominante del error de observación, expresada de forma polinomial en el domino de la variable compleja $s$, es:

$$
p_{1}(s)=s^{5}+l_{\theta 4} s^{4}+l_{\theta 3} s^{3}+l_{\theta 2} s^{3}+l_{\theta 1} s+l_{\theta 0} .
$$

Ahora, usando la ecuación (13) se propone el control basado en el observador GPI para el lazo desacoplado en el eje de Pitch. En este caso, las señales estimadas son entregadas por el observador de la ecuación (11).

$$
u_{\theta}(t)=\kappa_{\theta}(t)^{-1}\left[\ddot{\theta}_{r e f}(t)-k_{\theta 0}\left(\hat{\theta}(t)-\theta_{r e f}(t)\right)-k_{\theta 1}\left(\hat{\dot{\theta}}(t)-\dot{\theta}_{r e f}(t)\right)-\hat{\xi}_{\theta}(t)\right] .
$$

Considerando que el error de estimación $\epsilon_{\theta}(t)$ y sus derivadas son uniforme y absolutamente acotadas, es posible analizar la dinámica del error de seguimiento $E_{\theta}(t)=\theta(t)-\theta_{\text {ref }}(t)$. Esto se consigue si se incluye la ley de control de la ecuación (13) en el modelo simplificado y desacoplado de la ecuación (3), de manera que:

$$
p_{2}(s)=s^{2}+k_{\theta 1} s+k_{\theta 0} .
$$

Finalmente, los coeficientes $L_{\theta}=\left[\begin{array}{lllll}l_{\theta 4} & l_{\theta 3} & l_{\theta 2} & l_{\theta 1} & l_{\theta 0}\end{array}\right]^{T}$ pueden ser seleccionados a comodidad para satisfacer las condiciones deseadas para la dinámica del error de estimación. Asimismo, los coeficientes $k_{\theta 1}$ y $k_{\theta 0}$ en la ecuación (14) pueden seleccionarse para lograr la dinámica deseada del error de seguimiento en la inclinación del helicóptero 2-DOF. 


\section{Control lineal basado en observador GPI para el eje de Yaw}

Teniendo en cuenta los resultados de la sección anterior, en este apartado se planea un controlador para el eje de Yaw. Para ello, se asume un modelo interno de la perturbación generalizada tal que $d^{2} \xi_{\psi}(t) / d t^{2}=0$. Esta condición se plantea de acuerdo a la variación que se espera tenga la perturbación generalizada, la cual incluye los efectos del acople con el otro eje. De esta forma, y asumiendo el error de estimación como $\epsilon_{\psi}(t)=$ $\psi(t)-\hat{\psi}(t)$, se propone el siguiente observador GPI para la estimación de la función de perturbación generalizada y las derivadas de la salida plana en el subsistema desacoplado del eje de Yaw:

$$
\left[\begin{array}{c}
\frac{d \hat{\psi}^{(t)}}{d t} \\
\frac{d \hat{\psi}(t)}{d t} \\
\frac{d \hat{\xi}_{\psi}(t)}{d t} \\
\frac{d \hat{\xi}_{\psi}(t)}{d t}
\end{array}\right]=\left[\begin{array}{llll}
0 & 1 & 0 & 0 \\
0 & 0 & 1 & 0 \\
0 & 0 & 0 & 1 \\
0 & 0 & 0 & 0
\end{array}\right]\left[\begin{array}{c}
\hat{\psi}(t) \\
\hat{\dot{\psi}}(t) \\
\hat{\xi}_{\psi}(t) \\
\hat{\dot{\xi}}_{\psi}(t)
\end{array}\right]+\kappa_{\psi}(t)\left[\begin{array}{l}
0 \\
1 \\
0 \\
0
\end{array}\right] u_{\psi}(t)+\left[\begin{array}{l}
l_{\psi 3} \\
l_{\psi 2} \\
l_{\psi 1} \\
l_{\psi 0}
\end{array}\right] \epsilon_{\psi}(t)
$$

En este caso, el vector de ganancia del observador es $L_{\psi}=\left[\begin{array}{llll}l_{\psi 3} & l_{\psi 2} & l_{\psi 1} & l_{\psi 0}\end{array}\right]^{T}$. De este modo, la dinámica dominante del error de observación, expresada de forma polinomial en el domino de la variable compleja $s$, es:

$$
p_{3}(s)=s^{4}+l_{\psi 3} s^{3}+l_{\psi 2} s^{2}+l_{\psi 1} s+l_{\psi 0} .
$$

Al igual que lo realizado para el eje de Pitch, ahora se propone la siguiente ley de control basado en observador GPI para el eje de Yaw:

$$
u_{\psi}(t)=\kappa_{\psi}(t)^{-1}\left[\ddot{\psi}_{r e f}(t)-k_{\psi 0}\left(\hat{\psi}(t)-\psi_{r e f}(t)\right)-k_{\psi 1}\left(\hat{\psi}(t)-\dot{\psi}_{r e f}(t)\right)-\hat{\xi}_{\psi}(t)\right]
$$

Si se remplaza la ecuación (17) en el modelo simplificado y desacoplado para el eje de Yaw presentado en la ecuación (4), la dinámica del error de seguimiento en la rotación de helicóptero, definido como $E_{\psi}(t)=\psi(t)-$ $\psi_{\text {ref }}$, estará dominada de forma aproximada por el siguiente polinomio:

$$
p_{4}(s)=s^{2}+k_{\psi 1} s+k_{\psi 0}
$$

En la ecuación (15) los coeficientes $L_{\psi}=\left[\begin{array}{llll}l_{\psi 3} & l_{\psi 2} & l_{\psi 1} & l_{\psi 0}\end{array}\right]^{T}$ pueden ser seleccionados para satisfacer las especificaciones deseadas para la dinámica del error de estimación. Igualmente, los coeficientes $k_{\psi 1}$ y $k_{\psi 0}$ en la ecuación (18) pueden seleccionarse para lograr la dinámica deseada del error de seguimiento en la orientación del helicóptero 2-DOF.

\section{IDENTIFICACIÓN ALGEBRAICA DE PARÁMETROS EN EL HELICÓPTERO 2-DOF}

En esta sección se presenta una propuesta para la estimación de parámetros del helicóptero 2-DOF usando la metodología algebraica en el dominio del tiempo. Esta técnica de identificación se basa en el uso de manipulaciones algebraicas del modelo matemático del sistema usando tres pasos fundamentales: (i) la derivación en el dominio del tiempo de las ecuaciones, a fin de eliminar perturbaciones estructuradas (en los casos en que sea necesario); (ii) la multiplicación por potencias del tiempo y la realización de integraciones iteradas, a fin de eliminar las condiciones iniciales y obtener ecuaciones libres de derivadas explicitas; y (iii) la organización de las expresiones resultantes en términos de ecuaciones lineales en los parámetros (SiraRamírez et al., 2014a).

Como elemento novedoso, en este trabajo se incorpora el uso de los observadores GPI presentados en la sección anterior, con el fin de proveer una estimación de las derivadas de las salidas del sistema para su cálculo directo. Esto es importante en los casos donde existen operaciones no-lineales de difícil manipulación analítica desde la perspectiva del método algebraico clásico. Teniendo en cuenta estas consideraciones, durante el resto de esta sección se asume la siguiente notación simplificada para la integración iterativa en el dominio del tiempo:

$$
\int^{(p)} \phi(t)=\int_{t_{0}}^{(p)} \phi(t)=\int_{t_{0}}^{t} \int_{t_{0}}^{\sigma_{1}} \ldots \int_{t_{0}}^{\sigma_{p-1}} \phi\left(\sigma_{p}\right) d \sigma_{p} \ldots d \sigma_{1}, \text { con } t_{0}=0 .
$$




\section{Identificación de parámetros asociados al eje Pitch}

Con el propósito de identificar los parámetros $K_{p p}, K_{p y}, B_{p}$ y $F_{c p}$ asociados a la ecuación no-lineal de movimiento en Pitch es posible reescribir la ecuación (3) en la forma que se presenta en la ecuación (20), cuyos parámetros asociados son definidos en la Tabla 2.

$$
\begin{aligned}
& \ddot{\theta}(t)+a_{\theta} \sin 2 \theta(t) \dot{\psi}(t)^{2}-b_{\theta} \cos 2 \theta(t) \dot{\psi}(t)^{2}+c_{\theta} \cos \theta(t)+d_{\theta} \sin \theta(t)= \\
& e_{\theta} V_{m p}(t)+f_{\theta} V_{m y}(t)-g_{\theta} \dot{\theta}(t)-h_{\theta} .
\end{aligned}
$$

Considerando que se conocen los parámetros generales $J_{e q p}, M_{\text {heli }}, l_{m c}, h, g$, los parámetros $a_{\theta}, b_{\theta}, c_{\theta}$ y $d_{\theta}$ pueden ser estimados. De esta manera, los esfuerzos de identificación se deben concentrar en la estimación de $e_{\theta}, f_{\theta}, g_{\theta}$ y $h_{\theta}$.

Tabla 2: Parámetros asociados a la ecuación de movimiento de Pitch

\begin{tabular}{|c|c|}
\hline Parámetros conocidos & Parámetros desconocidos \\
\hline$a_{\theta}=\kappa_{\theta} \cdot M_{\text {heli }} \cdot\left(l_{m c}{ }^{2}-h^{2}\right) / 2$ & $e_{\theta}=\kappa_{\theta} \cdot K_{p p}$ \\
\hline$b_{\theta}=\kappa_{\theta} \cdot M_{\text {heli }} \cdot l_{m c} \cdot h$ & $f_{\theta}=\kappa_{\theta} \cdot K_{p y}$ \\
\hline$c_{\theta}=\kappa_{\theta} \cdot M_{\text {heli }} \cdot g \cdot l_{m c}$ & $g_{\theta}=\kappa_{\theta} \cdot B_{p}$ \\
\hline$d_{\theta}=\kappa_{\theta} \cdot M_{\text {heli }} \cdot g \cdot h$ & $h_{\theta}=\kappa_{\theta} \cdot F_{c p}$ \\
\hline
\end{tabular}

Teniendo en cuenta el modelo matemático asumido para el helicóptero 2-DOF se descarta la aplicación de derivadas con respecto al tiempo sobre las ecuaciones del sistema. Esto debido a que se desea estimar el parámetro $h_{\theta}$ en conjunto con los demás. Adicionalmente, realizar derivadas sobre la ecuación atenuará considerablemente algunos de los términos y podría reducir el condicionamiento numérico del sistema (SiraRamírez et al., 2014a). Bajo estas condiciones, la ecuación (20) se multiplica por $(-t)^{2}$ y se integra dos veces con respecto al tiempo, tal y como se indica a continuación:

$$
\begin{aligned}
& \int^{(2)} t^{2} \ddot{\theta}(t)+\int^{(2)} t^{2} a_{\theta} \sin 2 \theta(t) \dot{\psi}(t)^{2}-\int^{(2)} t^{2} b_{\theta} \cos 2 \theta(t) \dot{\psi}(t)^{2}+\int^{(2)} t^{2} c_{\theta} \cos \theta(t)+ \\
& \int^{(2)} t^{2} d_{\theta} \sin \theta(t)=\int^{(2)} t^{2} e_{\theta} V_{m p}(t)+\int^{(2)} t^{2} f_{\theta} V_{m y}(t)-\int^{(2)} t^{2} g_{\theta} \dot{\theta}(t)-\int^{(2)} t^{2} h_{\theta} .
\end{aligned}
$$

En la ecuación (21) se observa que los términos que multiplican a los parámetros $a_{\theta}$ y $b_{\theta}$ son de difícil manipulación analítica (desde la perspectiva del método algebraico) debido a la multiplicación por $\dot{\psi}(t)^{2}$. Una alternativa para poder continuar con el proceso de identificación es usar una versión estimada de la señal $\dot{\psi}(t)$, denominada $\hat{\dot{\psi}}(t)$, la cual es provista por el observador GPI de la ecuación (15). De esta manera, al reescribir la ecuación (21) usando la señal $\hat{\dot{\psi}}(t)$ se tiene lo siguiente:

$$
\begin{aligned}
& \int^{(2)} t^{2} \ddot{\theta}(t)+\int^{(2)} t^{2} a_{\theta} \sin 2 \theta(t) \hat{\psi}(t)^{2}-\int^{(2)} t^{2} b_{\theta} \cos 2 \theta(t) \hat{\dot{\psi}}(t)^{2}+\int^{(2)} t^{2} c_{\theta} \cos \theta(t)+ \\
& \int^{(2)} t^{2} d_{\theta} \sin \theta(t)=\int^{(2)} t^{2} e_{\theta} V_{m p}(t)+\int^{(2)} t^{2} f_{\theta} V_{m y}(t)-\int^{(2)} t^{2} g_{\theta} \dot{\theta}(t)-\int^{(2)} t^{2} h_{\theta} .
\end{aligned}
$$

Efectuando los cálculos correspondientes, la ecuación (23) muestra una relación libre del uso explícito de derivadas, a la vez que la ecuación (24) presenta su versión simplificada:

$$
\begin{aligned}
& t^{2} \theta(t)-4 \int^{(1)} t \theta(t)+2 \int^{(2)} \theta(t)+a_{\theta} \int^{(2)} t^{2} \sin 2 \theta(t) \hat{\dot{\psi}}(t)^{2}-b_{\theta} \int^{(2)} t^{2} \cos 2 \theta(t) \hat{\dot{\psi}}(t)^{2}+ \\
& c_{\theta} \int^{(2)} t^{2} \cos \theta(t)+d_{\theta} \int^{(2)} t^{2} \sin \theta(t)=e_{\theta} \int^{(2)} t^{2} V_{m p}(t)+f_{\theta} \int^{(2)} t^{2} V_{m y}(t)-g_{\theta}\left[\int^{(1)} t^{2} \theta(t)-\right. \\
& \left.2 \int^{(2)} t \theta(t)\right]-h_{\theta} \int^{(2)} t^{2} . \\
& q_{\theta}(t)=\left[\begin{array}{lllll}
p_{\theta 1,1}(t) & p_{\theta 1,2}(t) & p_{\theta 1,3}(t) & p_{\theta 1,4}(t)
\end{array}\right] \cdot\left[\begin{array}{llll}
e_{\theta} & f_{\theta} & g_{\theta} & h_{\theta}
\end{array}\right]^{T},
\end{aligned}
$$

Donde:

$$
\begin{aligned}
q_{\theta}(t)= & t^{2} \theta(t)-4 \int^{(1)} t \theta(t)+2 \int^{(2)} \theta(t)+a_{\theta} \int^{(2)} t^{2} \sin 2 \theta(t) \hat{\dot{\psi}}(t)^{2}-b_{\theta} \int^{(2)} t^{2} \cos 2 \theta(t) \hat{\dot{\psi}}(t)^{2}+ \\
& +c_{\theta} \int^{(2)} t^{2} \cos \theta(t)+d_{\theta} \int^{(2)} t^{2} \sin \theta(t)
\end{aligned}
$$




$$
\begin{aligned}
& p_{\theta 1,1}(t)=\int^{(2)} t^{2} V_{m p}(t) \\
& p_{\theta 1,2}(t)=\int^{(2)} t^{2} V_{m y}(t) \\
& p_{\theta 1,3}(t)=-\left[\int^{(1)} t^{2} \theta(t)-2 \int^{(2)} t \theta(t)\right] \\
& p_{\theta 1,4}(t)=-\int^{(2)} t^{2}
\end{aligned}
$$

De esta forma, una representación vectorial de la ecuación (24) será:

$$
q_{\theta}(t)=P_{\theta} \Theta_{\theta}, \quad \text { donde } P_{\theta}=\left[\begin{array}{lllll}
p_{\theta 1,1}(t) & p_{\theta 1,2}(t) & p_{\theta 1,3}(t) & p_{\theta 1,4}(t)
\end{array}\right] \text { y } \Theta_{\theta}=\left[\begin{array}{llll}
e_{\theta} & f_{\theta} & g_{\theta} & h_{\theta}
\end{array}\right]^{T},
$$

Una forma de obtener una solución para el vector de parámetros $P_{\theta}$ es realizando una multiplicación previa de ambos términos de la ecuación (26) por el vector columna $P_{\theta}{ }^{T}$, e integrar durante un intervalo de tiempo arbitrario $[0, t]$, de manera que:

$$
\int_{0}^{t} P_{\theta}^{T}(\lambda) q_{\theta}(\lambda) d \lambda=\left[\int_{0}^{t} P_{\theta}^{T}(\lambda) P_{\theta}(\lambda) d \lambda\right] \Theta_{\theta}
$$

La matriz $M_{p p_{-} \theta}=\int_{0}^{t} P_{\theta}^{T}(\lambda) P_{\theta}(\lambda) d \lambda$ (lado derecho de la ecuación (27)) resulta ser una matriz simétrica y positiva semidefinida para cualquier intervalo $t \geq \epsilon \geq 0$. Adicionalmente, se debe tener en cuenta que la matriz $M_{p p_{-} \theta}$ es invertible para un tiempo $(t)$ estrictamente positivo (Sira-Ramírez et al., 2014a). Esto último bajo las condiciones en las cuales el sistema es suficientemente bien condicionado desde el punto de vista numérico. Finalmente, efectuado operaciones algebraicas se obtiene la siguiente solución para el vector de parámetros estimados:

$$
\Theta_{\theta}=\left[\int_{0}^{t} P_{\theta}^{T}(\lambda) P_{\theta}(\lambda) d \lambda\right]^{-1} \cdot\left[\int_{0}^{t} P_{\theta}^{T}(\lambda) q_{\theta}(\lambda) d \lambda\right] .
$$

\section{Identificación de parámetros asociados al eje Yaw}

Siguiendo un proceso similar al descrito en el apartado anterior (metodología algebraica en el dominio del tiempo), es posible desarrollar un procedimiento para identificar los parámetros $K_{y p}, K_{y y}, B_{y}$ y $F_{c y}$ asociados a la ecuación no-lineal de movimiento en el eje Yaw. Para este caso, realizando el tratamiento correspondiente para la ecuación (2), que incluye la multiplicación por $(-t)^{2}$, la doble integración con respecto al tiempo y el uso de las variables estimadas $\hat{\dot{\theta}}(t)$ y $\hat{\dot{\psi}}(t)$, se consigue la siguiente expresión:

$$
q_{\psi}(t)=P_{\psi} \Theta_{\psi} \text {, donde } P_{\psi}=\left[\begin{array}{llll}
p_{\psi 1,1}(t) & p_{\psi 1,2}(t) & p_{\psi 1,3}(t) & p_{\psi 1,4}(t)
\end{array}\right] \quad \text { y } \Theta_{\psi}=\left[\begin{array}{llll}
d_{\psi} & e_{\psi} & f_{\psi} & g_{\psi}
\end{array}\right]^{T},
$$

Donde:

$$
\begin{aligned}
& q_{\psi}(t)=a_{\psi}\left[t^{2} \psi(t)-4 \int^{(1)} t \psi(t)+2 \int^{(2)} \psi(t)\right]+b_{\psi}\left[t^{2} \cos ^{2} \theta(t) \psi(t)-4 \int^{(1)} t \cos ^{2} \theta(t) \psi(t)+\right. \\
& \left.\int^{(1)} t^{2} \sin 2 \theta(t) \hat{\dot{\theta}}(t) \psi(t)+2 \int^{(2)} \cos ^{2} \theta(t) \psi(t)-2 \int^{(2)} t \sin 2 \theta(t) \hat{\dot{\theta}}(t) \psi(t)\right]+c_{\psi}\left[t^{2} \sin 2 \theta(t) \psi(t)-\right. \\
& \left.4 \int^{(1)} t \sin 2 \theta(t) \psi(t)-2 \int^{(1)} t^{2}(\cos 2 \theta) \hat{\dot{\theta}}(t) \psi(t)+2 \int^{(2)} \sin 2 \theta(t) \psi(t)+4 \int^{(2)} t \cos 2 \theta(t) \hat{\dot{\theta}}(t) \psi(t)\right] \\
& p_{\psi 1,1}(t)=\int^{(2)} t^{2} V_{m p}(t) \\
& p_{\psi 1,2}(t)=\int^{(2)} t^{2} V_{m y}(t) \\
& p_{\psi 1,3}(t)=-\left[\int^{(1)} t^{2} \psi(t)-2 \int^{(2)} t \psi(t)\right] \\
& p_{\psi 1,4}(t)=-\int^{(2)} t^{2}
\end{aligned}
$$

De esta manera, después de emplear los resultados obtenidos en el apartado anterior, se obtiene la siguiente solución para el vector de parámetros estimados en el eje de Yaw:

$$
\Theta_{\psi}=\left[\int_{0}^{t} P_{\psi}^{T}(\lambda) P_{\psi}(\lambda) d \lambda\right]^{-1} \cdot\left[\int_{0}^{t} P_{\psi}^{T}(\lambda) q_{\psi}(\lambda) d \lambda\right]
$$

Finalmente, en la Tabla 3 se presentan las definiciones de los parámetros asociados a las ecuaciones (29) y (30). 
Tabla 3: Parámetros asociados a la ecuación de movimiento de Yaw

\begin{tabular}{|c|c|}
\hline Parámetros conocidos & Parámetros desconocidos \\
\hline$a_{\psi}=J_{e q y}+\left(M_{\text {heli }} \cdot h^{2}\right)$ & $d_{\psi}=K_{y p}$ \\
\hline$b_{\psi}=M_{\text {heli }} \cdot\left(l_{m c}^{2}-h^{2}\right)$ & $e_{\psi}=K_{y y}$ \\
\hline$c_{\psi}=M_{\text {heli }} \cdot l_{m c} \cdot h$ & $f_{\psi}=B_{y}$ \\
\hline & $g_{\psi}=F_{c y}$ \\
\hline
\end{tabular}

\section{Criterios para la selección de parámetros estimados}

Con el propósito de evaluar el grado de ajuste de los parámetros estimados al modelo del helicóptero 2-DOF, en este trabajo se adoptó el criterio de la integral del cuadrado del error y el correspondiente índice de error. Este índice y el análisis de la sensibilidad a la variación de los parámetros fueron discutidos y analizados por Chiasson (2005) en la identificación de parámetros de máquinas eléctricas a través de la metodología de mínimos cuadrados. Por otra parte, Sira-Ramírez et al. (2014a) adoptó esta metodología dentro de un esquema de identificación en línea usando métodos algebraicos. En la Tabla 4 se resumen los criterios adoptados para la selección de los parámetros estimados en relación con los elementos definidos en las ecuaciones (26) y (29).

Tabla 4: Parámetros asociados a la ecuación de movimiento de Yaw

\begin{tabular}{|l|l|}
\hline \multicolumn{1}{|c|}{ Concepto } & \multicolumn{1}{|c|}{ Definición matemática } \\
\hline Error de estimación $\epsilon(\Theta, t)$ & $\epsilon(\Theta, t)=P(t) \Theta(t)-q(t)$ \\
\hline $\begin{array}{l}\text { Criterio de optimización basado en la integral } \\
\text { del cuadrado del error }\end{array}$ & $\begin{array}{l}\mathcal{J}(\Theta, t)=\frac{1}{2} \int_{0}^{t} \epsilon^{2}(\Theta, \lambda) d \lambda \\
(\text { Siendo } \mathcal{J}(\Theta, t) \text { la función de costo a minimizar) }\end{array}$ \\
\hline $\begin{array}{l}\text { Índice de error relativo }\left(I_{e}\right) . \\
\text { Aplica para un conjunto de parámetros }\end{array}$ & $I_{e}=\sqrt{\frac{M_{q q}-\left(M_{q p} \cdot M_{p p}{ }^{-1} \cdot M_{q p}{ }^{T}\right)}{M_{q q}}} \leq 1$ \\
\hline $\begin{array}{l}\text { Índice de error paramétrico (sensibilidad) } \\
\text { para } \mathcal{J}(\hat{\Theta}+\Delta \Theta)=2 \mathcal{J}(\hat{\Theta}, t) . \\
\text { Aplica para un parámetro en particular }\end{array}$ & $\begin{array}{l}I_{e p}=\Delta \Theta_{i}=\sqrt{\mathcal{J}(\hat{\Theta}, t) *\left[M_{p p(i, i)}\right]^{-1}} \\
M_{p p(i, i)} \operatorname{denota~el~elemento~}(i, i) \text { de la matriz } M_{p p} .\end{array}$ \\
\hline $\begin{array}{l}\text { Elementos de cálculo } \\
M_{q p} \epsilon \mathfrak{R}^{m x m}, M_{p q} y M_{q p} \epsilon \mathfrak{R} \text { y } M_{q q} \epsilon \mathfrak{R} \\
\text { donde } \mathrm{m} \text { es el número de parámetros a } \\
\text { estimar }\end{array}$ & $M_{p p} \triangleq \int_{0}^{t} P^{T}(\lambda) P(\lambda) d \lambda \quad M_{q p} \triangleq \int_{0}^{t} q(\lambda) P(\lambda) d \lambda$ \\
\end{tabular}

En relación al índice de error relativo $\left(I_{e}\right)$, se puede establecer que, para estimaciones fiables, éste debería ser mucho más pequeño que la unidad para cualquier conjunto de estimaciones. A su vez, si $I_{e}$ es muy cercano a uno, es posible concluir que el modelo asumido para el sistema no es el más adecuado. Otro análisis relevante se relaciona con el grado de sensibilidad del error con respecto a la variación de la magnitud de cada parámetro. Este concepto se puede cuantificar mediante la inclusión de índices de error paramétrico también denominado índice de sensibilidad (Chiasson, 2005). De esta manera, un índice de error paramétrico $\left(\Delta \Theta_{i}\right)$ pequeño indica que el error es muy sensible a cambios en el valor del parámetro estimado y, por lo tanto, se puede considerar que la calidad de la estimación es buena. Finalmente, un índice de error paramétrico grande indica que el parámetro estimado puede variar considerablemente sin un cambio significativo en el error, haciendo que el valor estimado sea poco confiable (Chiasson, 2005).

\section{RESULTADOS EXPERIMENTALES}

Durante el caso de estudio se planeó un experimento de identificación algebraica en lazo cerrado. En este ejercicio, todas las pruebas se realizaron bajo la acción del control lineal multivariable basado en observadores GPI (descrito anteriormente) y en un escenario libre de perturbaciones externas. Los parámetros iniciales para el controlador son $K_{p p}=0.0279, K_{p y}=0.0029, K_{y p}=0.0005$ y $K_{y y}=0.0525$, los cuales fueron tomados de la información entregada por el fabricante (Quanser inc., 2012). En este sentido, la incertidumbre presente en estos parámetros será tolerada por el controlador propuesto dada sus características de robustez. Para la implementación de los controladores se usó el software para control en tiempo real sobre Windows, WinCon ${ }^{\mathrm{TM}}$ de Quanser inc. $\AA^{\circledR}$, con el cual se compilan los diagramas desarrollados en Simulink de Matlab®, empleando el método de integración de Runge-Kuta y un periodo de muestreo de $1 \mathrm{~ms}$. Por otra parte, las señales desde y hacia el exterior son administradas por la tarjeta de adquisición MultiQ4-PCI de Quanser inc.®. Las características generales de las pruebas se presentan en la Tabla 5 y los resultados de las pruebas se muestran en las Figuras 1 a 4. 


\section{Resultados de la identificación de parámetros}

La Figura 1 (fila superior) muestra el índice de error relativo $\left(I_{e_{-}}\right.$itch $)$asociado al vector $\Theta_{\theta}$. Este vector contiene la estimación de los parámetros desconocidos de la ecuación de movimiento del eje de Pitch. Usar este índice permite evaluar el grado ajuste del modelo estimado con respecto al comportamiento real en la inclinación del helicóptero 2-DOF, lo cual se logra a medida que el identificador algebraico de la ecuación (28) obtiene el conjunto de parámetros estimados. De esta manera, al localizar el valor mínimo de $I_{e_{-} p i t c h}$, es posible determinar el instante de tiempo en el cual el modelo propuesto (ver ecuación (1)) predice con mayor precisión el comportamiento real del sistema en eje de Pitch. En este caso, la prueba experimental tuvo una duración de 80 segundos y el menor índice de error relativo, para el conjunto de parámetros $\Theta_{\theta}$, ocurre en 46.12 segundos (punto identificado con color verde). En consecuencia, una vez identificado dicho instante, se toman los valores correspondientes a los parámetros $K_{p p}, K_{p y}, B_{p}$ y $F_{c p}$ (valores estimados).

Tabla 5: Características generales de las pruebas

\begin{tabular}{|l|l|l|}
\hline \multicolumn{1}{|c|}{ Elemento } & \multicolumn{1}{|c|}{ Inclinación (Pitch) } & \multicolumn{1}{c|}{ Orientación (Yaw) } \\
\hline Señales de referencia & $\theta_{\text {ref }}(t)=-10^{\circ}+20^{\circ} \sin (0.1 t+3 \pi / 2)$ & $\psi_{\text {ref }}(t)=20^{\circ}+15^{\circ} \sin (0.1 t+3 \pi / 2)$ \\
\hline $\begin{array}{l}\text { Diseño de Observadores } \\
\text { GPI }\end{array}$ & $\begin{array}{l}p_{1}(s)=(s+w)\left(s^{2}+2 z w s+w^{2}\right)^{2} \\
\operatorname{con} w=12 \mathrm{y} z=7\end{array}$ & $\begin{array}{l}p_{3}(s)=\left(s^{2}+2 z w s+w^{2}\right)^{2} \\
\operatorname{con} w=10 \mathrm{y} z=1.2\end{array}$ \\
\hline Diseño de controladores & $\begin{array}{l}p_{2}(s)=s^{2}+2 z w s+w^{2} \\
\operatorname{con} w=0.5 \mathrm{y} z=1\end{array}$ & $\begin{array}{l}p_{4}(s)=s^{2}+2 z w s+w^{2} \\
\operatorname{con} w=0.65 \mathrm{y} z=1\end{array}$ \\
\hline Condición inicial & $\theta(0)=-40.5^{\circ}$ & $\psi(0)=0^{\circ}$ \\
\hline
\end{tabular}

La Figura 1 también presenta los índices de sensibilidad correspondientes a cada parámetro estimado del vector $\Theta_{\theta}$ (filas 2 y 3 ). La comparación entre las magnitudes de los índices de sensibilidad $\left(\Delta K_{p p}=3.602 \times\right.$ $10^{-3}, \Delta K_{p y}=2.971 \times 10^{-4}, \Delta B_{p}=9.868 \times 10^{-3}$ y $\left.\Delta F_{c p}=5.289 \times 10^{-2}\right)$, permite establecer que los parámetros asociados a las contantes de voltaje a par $\left(K_{p y}\right.$ y $\left.K_{p p}\right)$ son identificados con mayor precisión que los parámetros asociados a la fricción viscosa y a la fricción de Coulomb $\left(B_{p}\right.$ y $\left.F_{c p}\right)$. Lo anterior considerando que $K_{p y}$ y $K_{p p}$ tienen índices de sensibilidad menores dentro del vector $\Theta_{\theta}$.
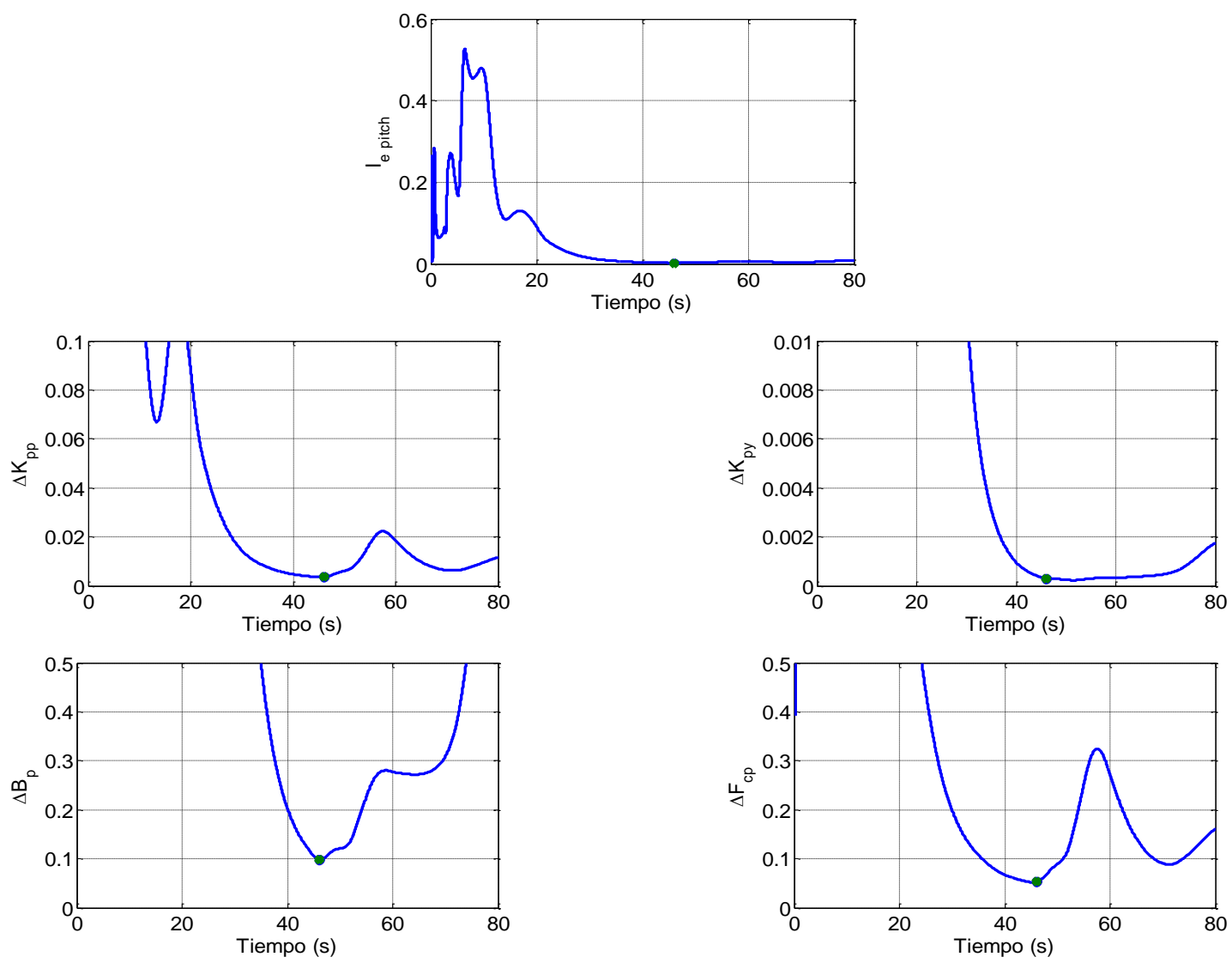

Fig .1: Índice de error relativo (fila 1) e índices de sensibilidad (filas 2 y 3) en el eje Pitch 
La Figura 2 muestra la estimación obtenida para los parámetros $K_{p p}, K_{p y}, B_{p}$ y $F_{c p}$, los cuales están asociados a ecuación de movimiento en el eje de Pitch. Es posible apreciar que al iniciar las pruebas todos los parámetros tardan un tiempo aproximado de 23 segundos en converger a valores confiables. Este tiempo de transición es debido al tiempo de asentamiento que requiere el observador GPI (ecuación (15)) para el cálculo del identificador algebraico (ecuación (28)). Dicha transición también se debe a características propias de la prueba experimental tales como: el efecto del ruido, el condicionamiento numérico del sistema, la persistencia de la excitación y el tiempo de procesamiento de los algoritmos computacionales.

Dado que el propósito de este trabajo es obtener un modelo del helicóptero 2-DOF con parámetros constantes (únicos), los valores de la estimación que registraron el índice de sensibilidad mínimo (de acuerdo a los resultados presentados en la Figura 1) serán los valores seleccionados para el modelo final (puntos de color verde en la Figura 2). Considerando lo anterior, los valores obtenidos son: $K_{p p}=2.559 \times 10^{-2} \mathrm{~N} \cdot \mathrm{m} / \mathrm{V}, K_{p y}=$ $2.555 \times 10^{-3} \mathrm{~N} \cdot \mathrm{m} / \mathrm{V}, B_{p}=5.495 \times 10^{-2} \mathrm{Kg} \cdot \mathrm{m}^{2} / \mathrm{s}, F_{c p}=-1.968 \times 10^{-1} \mathrm{~N} \cdot \mathrm{m}$.
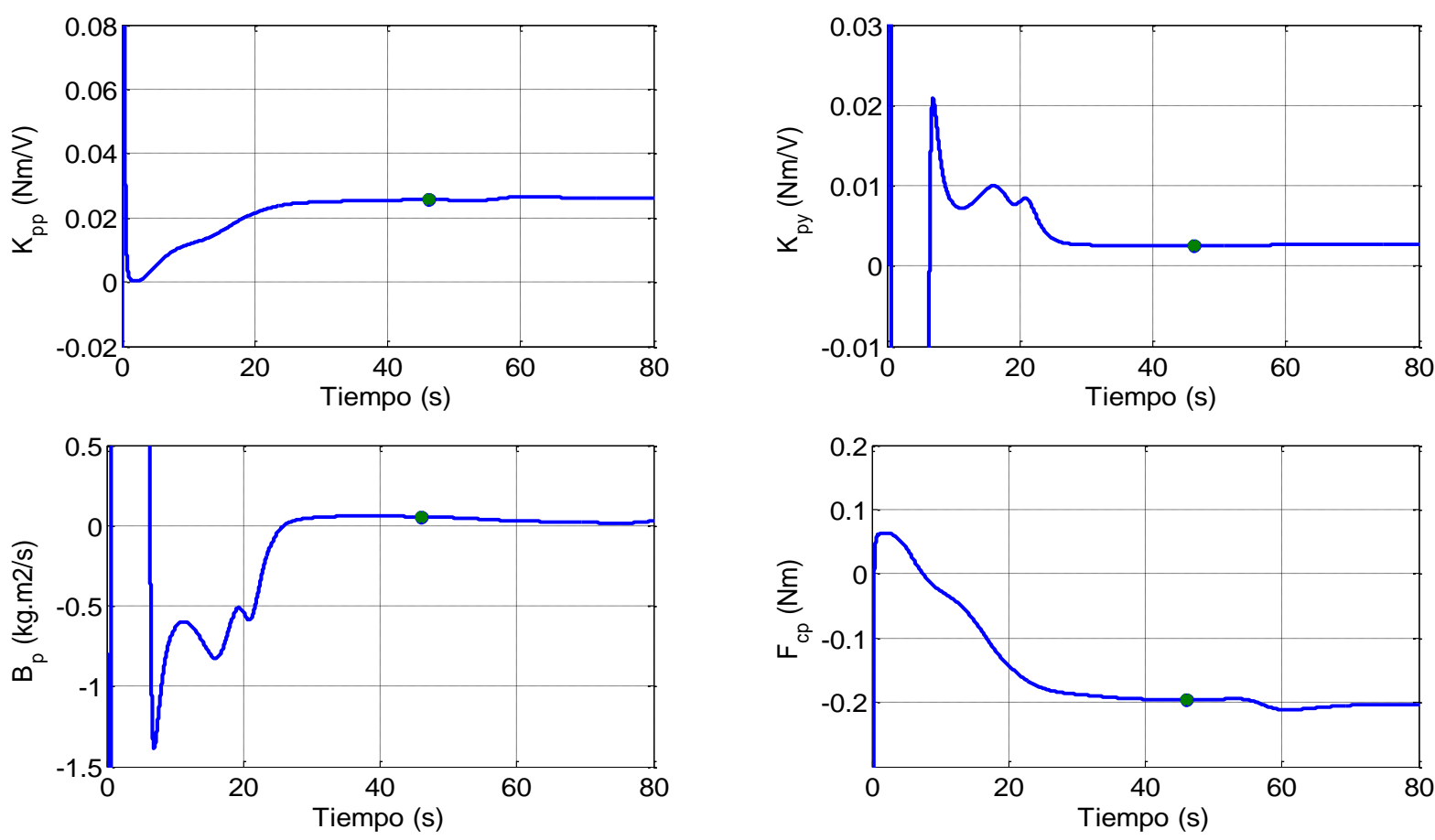

Fig 2: Identificación algebraica de los parámetros estimados en el eje Pitch

Por otra parte, la Figura 3 (fila superior) ilustra el comportamiento en el tiempo del índice de error relativo $\left(I_{e_{-} \text {yaw }}\right)$ asociado al vector $\Theta_{\psi}$. Este vector incluye la estimación de los parámetros desconocidos en la ecuación de movimiento del eje de Yaw. Para este caso, la prueba experimental también duró 80 segundos y el menor índice de error $\left(I_{e_{-} \text {yaw }}\right)$ ocurrió en 66.68 segundos (resaltado en color verde). Dado que en dicho instante de tiempo el modelo propuesto (ecuación (2)) y los parámetros obtenidos predicen de una manera más precisa el comportamiento real del sistema en eje de Yaw, es posible tomar en dicho punto los valores de los parámetros $K_{y p}, K_{y y}, B_{y}$ y $F_{c y}$.

Adicionalmente, en la Figura 3 (filas 2 y3) se presentan los índices de sensibilidad obtenidos durante la estimación para cada parámetro del vector $\Theta_{\psi}$. La comparación entre las magnitudes de los índices de sensibilidad $\left(\Delta K_{y p}=1.334 \times 10^{-5}, \Delta K_{y y}=4.028 \times 10^{-4}, \Delta B_{y}=2.737 \times 10^{-2}\right.$ y $\left.\Delta F_{c y}=2.521 \times 10^{-3}\right)$ muestra que los parámetros relacionados con las contantes de voltaje a par $\left(K_{y p}\right.$ y $\left.K_{y y}\right)$ presentan índices de sensibilidad más bajos que los registrados para a la fricción viscosa y a la fricción de Coulomb $\left(B_{y}\right.$ y $\left.F_{c y}\right)$. Esto permite identificar con mayor precisión los parámetros $K_{y p}$ y $K_{y y}$.

Para complementar estos resultados, la Figura 4 muestra la estimación de los parámetros $K_{y p}, K_{y y}, B_{y}$ y $F_{c y}$, asociados a la ecuación de movimiento en el eje de Yaw. En este caso, los valores que registraron el mínimo índice de sensibilidad, de acuerdo la Figura 3, serán los valores seleccionados para el modelo final del helicóptero 2-DOF. De esta forma, los parámetros obtenidos son: $K_{y p}=1.019 \times 10^{-3} \mathrm{~N} \cdot \mathrm{m} / \mathrm{V}, K_{y y}=1.853 \times$ $10^{-2} \mathrm{~N} \cdot \mathrm{m} / \mathrm{V}, B_{y}=1.316 \mathrm{Kg} \cdot \mathrm{m}^{2} / \mathrm{s}, F_{c y}=9.363 \times 10^{-2} \mathrm{~N} \cdot \mathrm{m}$. 
Al igual que en la estimación de los parámetros para el eje de Yaw, es posible apreciar que al iniciar el experimento los parámetros tardan un tiempo en converger a valores confiables, Este tiempo de transición (40 segundos aprox.) es debido a las características propias del experimento (mencionadas en el análisis de los resultados en el eje Pitch) y al tiempo de asentamiento que requieren los observadores GPI descritos en las ecuaciones (11) y (15), los cuales proveen las señales $\hat{\theta}(t)$ y $\hat{\psi}(t)$ indispensables para el cálculo del identificador algebraico presentado en la ecuación (31).
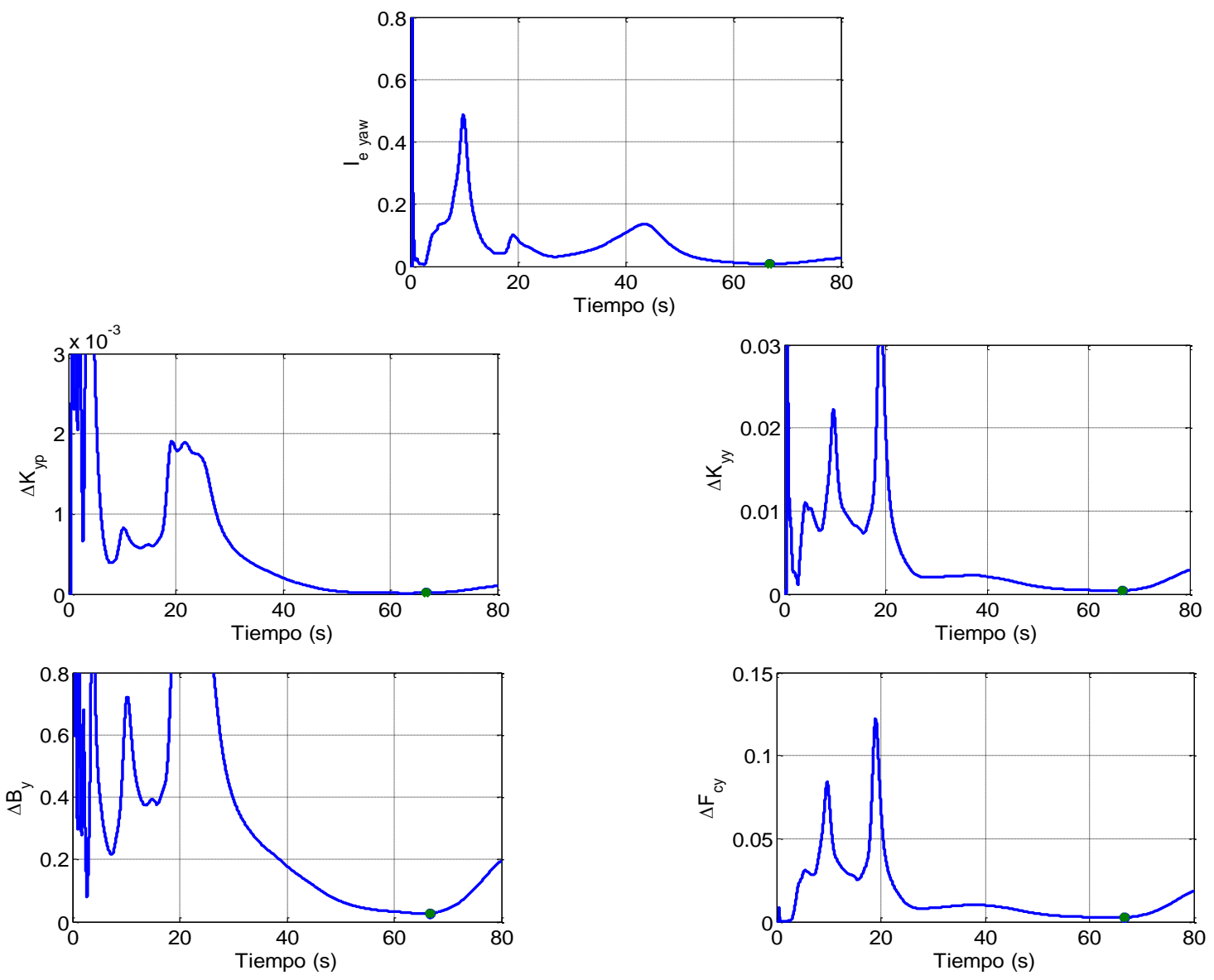

Fig 3: Índice de error relativo (fila 1) e índices de sensibilidad (filas 2 y 3) en el eje Yaw
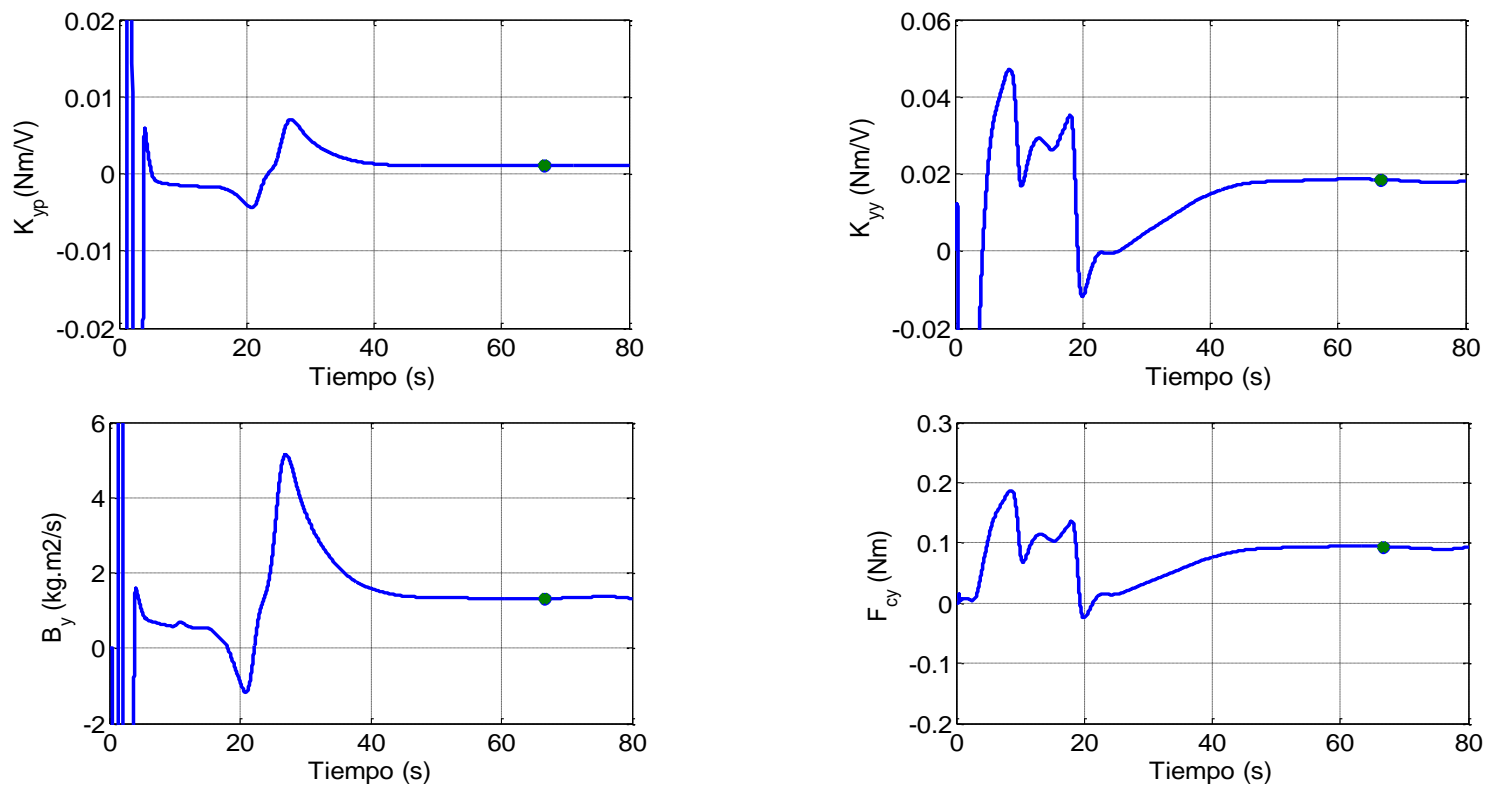

Fig. 4: Identificación algebraica de los parámetros estimados en el eje Yaw 


\section{Validación de los parámetros obtenidos durante la estimación}

Una alternativa para validar los parámetros obtenidos durante las estimaciones es comparar los resultados experimentales del helicóptero 2-DOF bajo control (mediciones reales) y la simulación del modelo matemático propuesto, el cual incorpora el conjunto de parámetros estimados. Las características de las pruebas realizadas en ambos casos (simulación y experimentación) fueron presentadas en la tabla 5 y la duración de la misma fue ampliada a 125 segundos. Adicionalmente, para la simulación se utilizó el software Simulink de Matlab® con un periodo de muestreo de $1 \mathrm{~ms}$, se seleccionó el método de integración de Runge-Kuta y se incluyó el efecto aditivo del ruido en las posiciones angulares $(\theta$ y $\psi$ ) mediante procesos aleatorios uniformemente distribuidos con un valor máximo de 0.1 grados.

En la Figura 5 se presentan los resultados obtenidos en la validación usando tres señales de comparación: ( $I)$ las posiciones angulares $\theta$ y $\psi$; (ii) los voltajes aplicados a los motores $V_{m p}$ y $V_{m y}$; (iii) las perturbaciones generalizadas estimadas $\xi_{\theta}$ y $\xi_{\psi}$. En esta figura es posible distinguir dos regiones de operación del sistema: la primera correspondiente al arranque (entre 0 y 20 segundos), donde se aprecia el transitorio en la dinámica de rastreo de las posiciones angulares (fila 1) y algunos cambios bruscos en los voltajes de control (fila 2) y las estimaciones de las perturbaciones generalizadas (fila 3). Todos estos efectos son causados por la convergencia de los observadores y la diferencia entre la condición inicial de las salidas con respecto a las referencias. La segunda región, correspondiente a la operación nominal del sistema (desde 20 segundos y hasta el final de la prueba), está caracterizada por un buen seguimiento de la referencia y variaciones de menor amplitud en las señales de control.
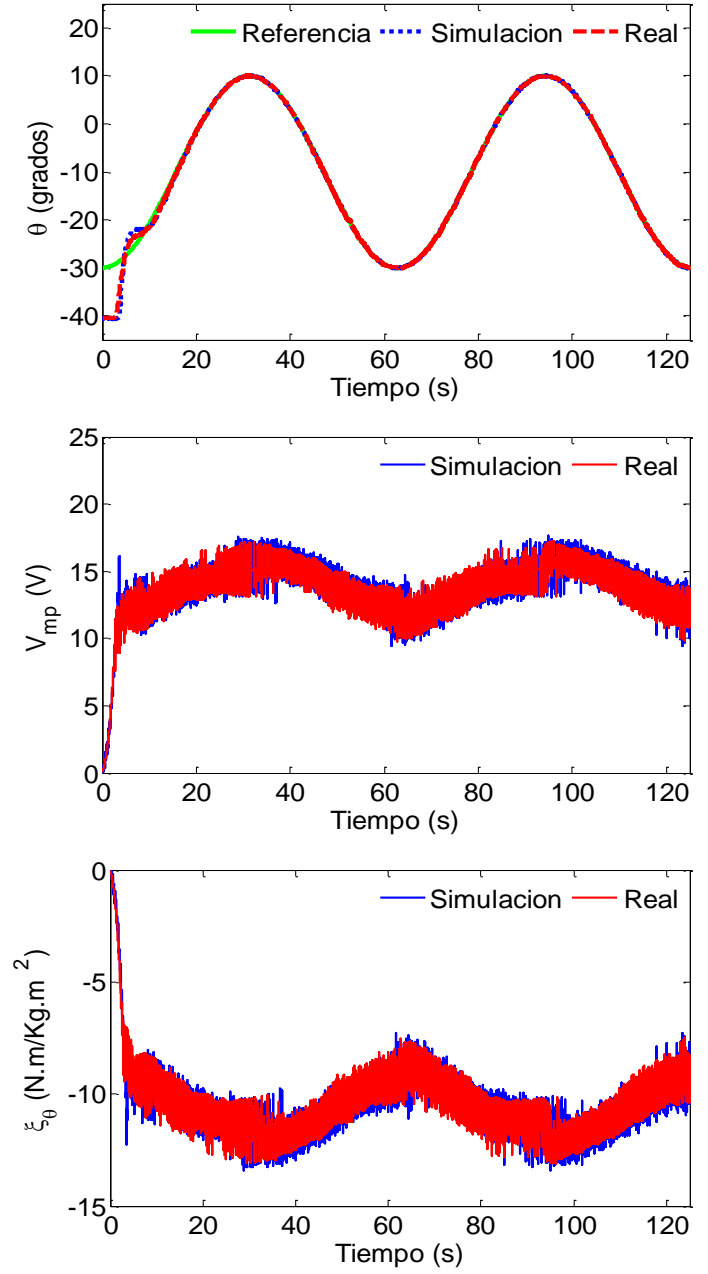
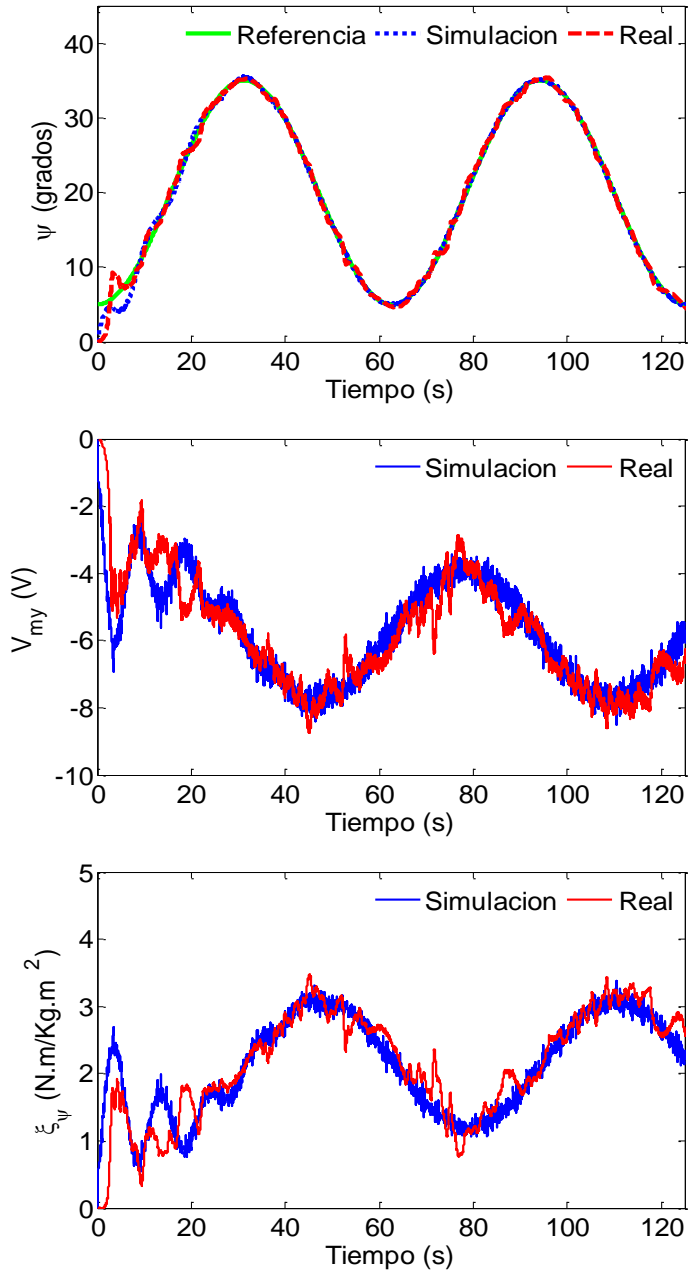

Fig. 5: Validación del modelo comparando simulaciones (línea azul) y mediciones reales (línea roja).

fila 1: ángulos -- fila 2: voltajes aplicados a los motores -- fila 3: perturbaciones generalizadas estimadas

En la Figura 5 también es posible apreciar que los voltajes aplicados (fila 2) y las perturbaciones generalizadas (fila 3) tienen algunas componentes de alta frecuencia (oscilaciones rápidas), las cuales se deben a las ganancias de los observadores GPI. Finalmente, dada la similitud observada entre los valores medios obtenidos en las simulaciones y las mediciones reales (para los voltajes aplicados y las perturbaciones 
generalizadas), es posible concluir que el modelo obtenido con parámetros únicos puede ser usado en aplicaciones generales con fines de control y diagnóstico. No obstante, para aplicaciones que demanden mayores desempeños, será necesario incorporar todo el esquema de identificación a fin de obtener parámetros variantes en el tiempo.

Para analizar con mayor nivel de detalle los resultados presentados en la Figura 5, en cada caso, se calculó la diferencia (error) entre las señales reales y las señales obtenidas a partir de la simulación. Asimismo, con el fin de reducir el efecto de las componentes de alta frecuencia en las señales, se procesaron los errores calculados con ayuda de un filtro pasa bajos de primer orden y un ancho de banda de $10 \mathrm{rad} / \mathrm{s}$. En la Figura 6 se muestran los resultados de esta comparación y los errores obtenidos para las posiciones angulares, los voltajes aplicados a los motores y las perturbaciones generalizadas estimadas.
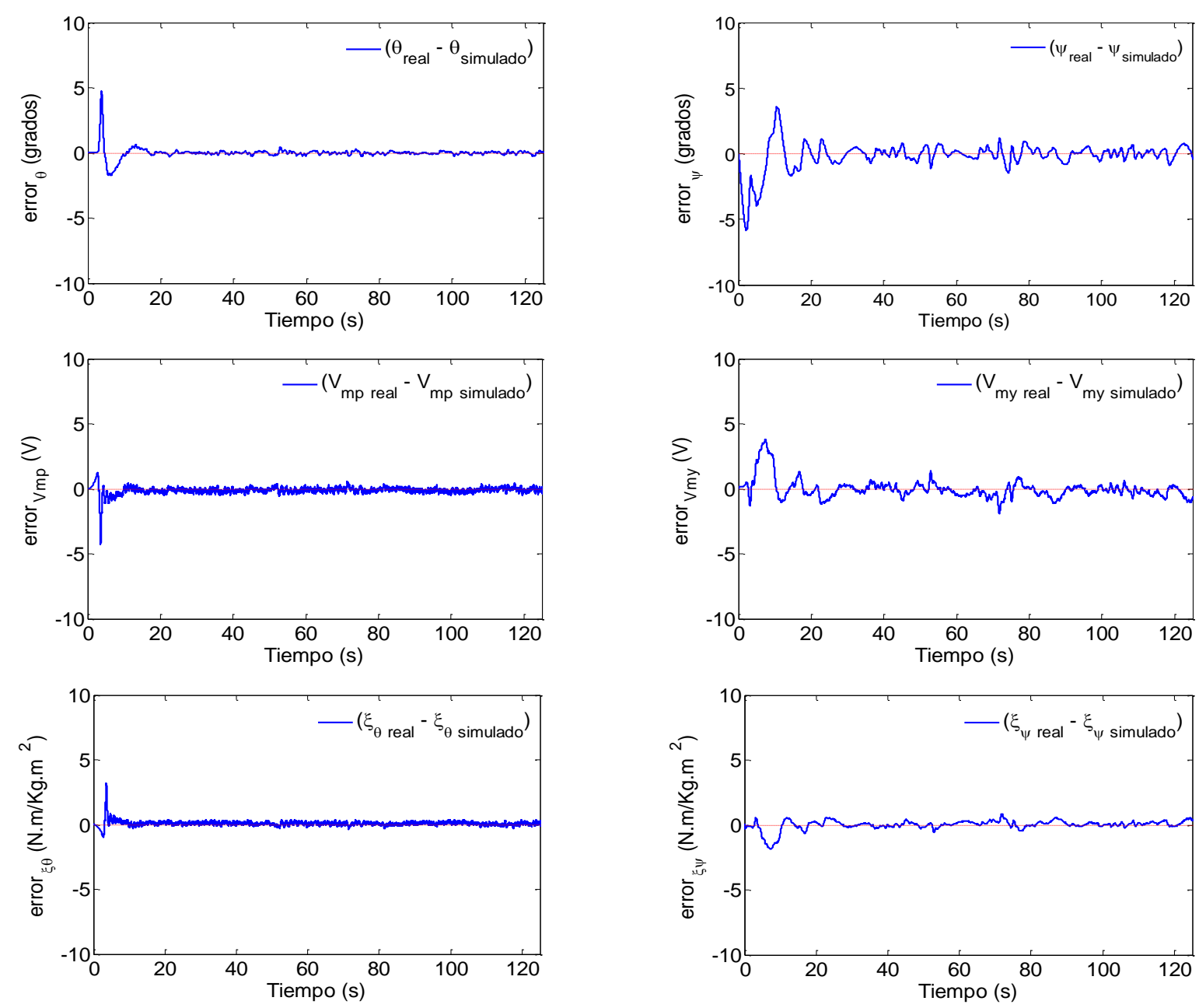

Fig. 6: Errores de validación (diferencia entre mediciones reales y simulaciones) para el modelo de estimación Error (línea azul) - Referencia en el punto cero (roja punteada)

En la Figura 6 se puede apreciar (para todos los casos) que los errores de mayor magnitud se presentan al iniciar la prueba, es decir, en el tiempo comprendido entre 0 y 20 segundos. Esto se debe a que el modelo con parámetros únicos no se ajusta con la misma precisión al comportamiento real del sistema en todas sus regiones de trabajo, especialmente en la zona de arranque del sistema. No obstante, superado el arranque, los errores se reducen considerablemente y mantienen valores medios cercanos a cero.

Otro aspecto a resaltar de los resultados presentados en la Figura 6, es que los errores de las señales correspondientes al eje de Yaw (columna de la derecha) son, en general, de mayor magnitud que los obtenidos en el eje de Pitch. Este comportamiento permite inferir que el modelo identificado para la inclinación (eje de Pitch), en comparación con su contraparte para la rotación (eje de Yaw), predice con mayor precisión el comportamiento real del sistema. Esta condición puede obedecer, por un lado, a la presencia de elementos no modelados en la dinámica que describe el movimiento en el eje de Yaw (ecuación 2) y, por otro lado, a características propias del experimento como el condicionamiento numérico y la persistencia de la excitación en dicho eje. 


\section{CONCLUSIONES}

En este artículo se propuso una alternativa para la estimación simultánea de un conjunto de ocho parámetros asociados al modelo no-lineal de un helicóptero de dos grados de libertad (2-DOF) que opera bajo control de lazo cerrado. La principal contribución de este trabajo radica en la presentación de una variante de la metodología algebraica para la estimación de parámetros, la cual involucra el uso de observadores proporcional integral generalizados (GPI). Estos observadores son los encargados de proveer una estimación de las derivadas de las salidas del sistema, para su cómputo directo, en los casos donde existen operaciones no-lineales de difícil manipulación analítica desde la perspectiva del método algebraico clásico.

Con el propósito de evaluar el grado de ajuste de los parámetros estimados al modelo del helicóptero 2-DOF, se adoptó el criterio de la integral del cuadrado del error y los correspondientes índices de error y de sensibilidad. Estos indicadores mostraron que los parámetros estimados con mayor precisión fueron los asociados a la relación de voltaje a par en los motores, en comparación con los coeficientes de fricción viscosa y el término asociado a la fricción de Coulomb.

El modelo que incluye los parámetros estimados fue validado por medio de simulaciones y realizando una comparación con mediciones reales. Esta comparación permitió establecer que existe un mejor ajuste en las variables asociadas al eje de Pitch (inclinación). Sin embargo, los resultados obtenidos para el modelo en conjunto son aceptables y habilitan el uso de los parámetros estimados para diferentes aplicaciones de diagnóstico y control.

\section{AGRADECIMIENTOS}

Los autores quieren extender su agradecimiento al Centro de Electricidad, Electrónica y Telecomunicaciones del SENA y al Sistema de Investigación, Desarrollo tecnológico e Innovación del SENA (SENNOVA) quienes financiaron la publicación de este trabajo.

\section{REFERENCIAS}

Becedas, J., J.R. Trapero, V. Feliu y H. Sira-Ramírez, Adaptive Controller for Single-Link Flexible Manipulators Based on Algebraic Identification and Generalized Proportional Integral Control, doi: 10.1109/TSMCB.2008.2008905, IEEE Transactions on Systems, Man, and Cybernetics, Part B (Cybernetics), 39(3), 735-751 (2009)

Beltrán-Carbajal, F. y G. Silva-Navarro, Adaptive-Like Vibration Control in Mechanical Systems with Unknown Paramenters and Signals, doi: 10.1002/asjc.727, Asian Journal of Control, 15(6), 1613-1626 (2013)

Beltrán-Carbajal, F., G. Silva-Navarro y L.G. Trujillo-Franco, On-Line Parametric Estimation of Damped Multiple Frequency Oscillations, doi: 10.1016/j.epsr.2017.09.013, Electric Power Systems Research, 154, 423-432 (2018)

Bougrine, M., M. Benmiloud y otros tres autores, Load Estimator-Based Hybrid Controller Design for Two-Interleaved Boost Converter Dedicated to Renewable Energy and Automotive Applications, doi: 10.1016/j.isatra.2016.09.001, ISA transactions, 66, 425-436 (2017)

Campuzano-Martínez, I.R., Diagnóstico de Generadores Eléctricos de Potencia con Técnicas de Monitoreo en Línea y Fuera de Línea, doi: 10.4067/S0718-07642016000200003, Información Tecnológica, 27(2), 11-20 (2016)

Chiasson, J., Modeling and High Performance Control of Electric Machines, 548-564, John Wiley \& Sons, New Jersey, United States (2005)

Cortés-Romero, J., H. Rojas-Cubides y otros tres autores, Active Disturbance Rejection Approach for Robust FaultTolerant Control via Observer Assisted Sliding Mode Control, doi: 10.1155/2013/609523, Mathematical Problems in Engineering, 1-13 (2013)

Cortés-Romero, J., G.A. Ramos y H. Coral-Enriquez, Generalized Proportional Integral Control for Periodic Signals under Active Disturbance Rejection Approach, doi: 10.1016/j.isatra.2014.07.001, ISA transactions, 53(6), 1901-1909 (2014)

Cortés-Romero, J., A. Jimenez-Triana, H. Coral-Enriquez y H. Sira-Ramírez, Algebraic Estimation and Active Disturbance Rejection in the Control of Flat Systems, doi: 10.1016/j.conengprac.2017.02.009, Control Engineering Practice, 61, 173$182(2017)$

Delpoux, R. y T. Floquet, On-line Parameter Estimation via Algebraic Method: An Experimental Illustration, doi: 10.1002/asjc.870, Asian Journal of Control, 17(1), 315-326 (2015)

Fliess, M. y H. Sira-Ramírez, An Algebraic Framework for Linear Identification, doi: 10.1051/cocv:2003008, ESAIM: Control, Optimization and Calculus of Variations, 9, 151-168 (2003)

Fliess, M., C. Join y H. Sira-Ramírez, Robust Residual Generation for Linear Fault Diagnosis: an Algebraic Setting with Examples, doi: 10.1080/002071704200024374, International Journal of Control, 77(14), 1223-1242 (2004)

Fliess, M., C. Join y H. Sira-Ramirez, Non-linear Estimation is Easy, doi: 10.1504/IJMIC.2008.020996, International Journal of Modelling, Identification and Control, 4(1), 12-27 (2008) 
Flores, R. y T.I. Asiaín, Diagnóstico de Fallas en Máquinas Eléctricas Rotatorias Utilizando la Técnica de Espectros de Frecuencia de Bandas Laterales, doi: 10.4067/S0718-07642011000400009, Información Tecnológica, 22(4), 73-84 (2011)

Garcia-Rodríguez, C., J. Cortés-Romero y H. Sira-Ramírez, Algebraic Identification and Discontinuous Control for Trajectory Tracking in a Perturbed 1-dof Suspension System, doi: 10.1109/TIE.2009.2026383, IEEE Transactions on Industrial Electronics, 56(9), 3665-3674 (2009)

Garrido, R. y A. Concha, An Algebraic Recursive Method for Parameter Identification of a Servo Model, doi: 10.1109/TMECH.2012.2208197, IEEE/ASME Transactions on Mechatronics, 18(5), 1572-1580 (2013)

Guo, B. y Z. Zhao, On Convergence of the Nonlinear Active Disturbance Rejection Control for MIMO Systems, doi: 10.1137/110856824, SIAM Journal on Control and Optimization, 51(2), 1727-1757 (2013)

Kiltz, L., C. Join, M. Mboup y J. Rudolph, Fault-Tolerant Control Based on Algebraic Derivative Estimation Applied on a Magnetically Supported Plate, doi: 10.1016/j.conengprac.2014.01.009, Control Engineering Practice, 26, 107-115 (2014)

Kodamana, H., B. Huang y otros cuatro autores, Approaches to Robust Process Identification: A Review and Tutorial of Probabilistic Methods, doi: 10.1016/j.jprocont.2018.02.011, Journal of Process Control, 66, 68-83 (2018)

Linares-Flores, J., A.H. Mendez, C. Garcia-Rodriguez y H. Sira-Ramirez, Robust Nonlinear Adaptive Control of a "Boost" Converter via Algebraic Parameter Identification, doi:10.1109/TIE.2013.2284150, IEEE Transactions on Industrial Electronics, 61(8), 4105-4114 (2014)

Luviano-Juarez, A., J. Cortes-Romero y H. Sira-Ramirez, Synchronization of Chaotic Oscillators by Means of Generalized Proportional Integral Observers, doi:10.1142/S0218127410026654, International Journal of Bifurcation and Chaos, 20(05), 1509-1517 (2010)

Martínez-Guerra, R., I. Trejo-Zúñiga y F. Meléndez-Vázquez, A Dynamical Controller with Fault-Tolerance: Real-Time Experiments, doi: 10.1016/j.jfranklin.2017.02.038, Journal of the Franklin Institute, 354(8), 3378-3404 (2017)

Menhour, L., B. D'Andréa-Novel, M. Fliess y H. Mounier, Coupled Nonlinear Vehicle Control: Flatness-Based Setting with Algebraic Estimation Techniques, doi: 10.1016/j.conengprac.2013.09.013, Control Engineering Practice, 22, 135-146 (2014)

Miranda-Colorado, R. y J. Moreno-Valenzuela, An Efficient On-Line Parameter Identification Algorithm for Nonlinear Servomechanisms with an Algebraic Technique for State Estimation, doi:10.1002/asjc.1511, Asian Journal of Control, 19, 2127-2142 (2017)

Morales, R., E. Segura y otros tres autores, Online Signal Filtering Based on the Algebraic Method and its Experimental Validation, doi:10.1016/j.ymssp.2015.06.021, Mechanical Systems and Signal Processing, 66, 374-387 (2016)

Nelles, O., Nonlinear System Identification: from Classical Approaches to Neural Networks and Fuzzy Models, 482-509, Springer Science \& Business Media, Berlin, Germany (2013)

Pereira, E., J.R. Trapero, I.M. Díaz y V. Feliu, Adaptive Input Shaping for Single-Link Flexible Manipulators using an Algebraic Identification, doi:10.1016/j.conengprac.2011.10.004, Control Engineering Practice, 20(2), 138-147 (2012)

Quanser Inc., User Manual 2 DOF Helicopter Experiment, Set Up and Configuration (2012)

Rojas-Cubides, H., J. Cortés-Romero y H. Rojas-Cubides, Control por Rechazo Activo de Perturbaciones Basado en Observadores GPI para un Helicóptero de Dos Grados de Libertad, Ingenio Magno, 6(1), 12-22 (2015)

San-Millan, A. y V. Feliu, A Fast Online Estimator of the Two Main Vibration Modes of Flexible Structures from Biased and Noisy Measurements, doi:10.1109/TMECH.2014.2304302, IEEE/ASME Transactions on Mechatronics, 20(1), 93-104 (2015)

Schoukens, M. y K. Tiels, Identification of Block-Oriented Nonlinear Systems Starting from Linear Approximations: a Survey, doi: 10.1016/j.automatica.2017.06.044, Automatica, 85, 272-292 (2017)

Sira-Ramírez, H., C. García-Rodríguez, J. Cortés-Romero y A. Luviano-Juárez, Algebraic Identification and Estimation Methods in Feedback Control Systems, 136-141, John Wiley \& Sons, United Kindom (2014 a)

Sira-Ramírez, H., J. Linares-Flores, C. García-Rodríguez y M. Contreras-Ordaz, On the Control of the Permanent Magnet Synchronous Motor: an Active Disturbance Rejection Control Approach, doi: 10.1109/TCST.2014.2298238, IEEE Transactions on Control Systems Technology, 22(5), 2056-2063 (2014 b)

Wang, W.X., Y.C. Lai y C. Grebogi, Data Based Identification and Prediction of Nonlinear and Complex Dynamical Systems, doi: 10.1016/j.physrep.2016.06.004, Physics Reports, 644, 1-76 (2016)

Zurita-Bustamante, E., J. Linares-Flores, E. Guzmán-Ramírez y H. Sira-Ramírez, A Comparison Between the GPI and PID Controllers for the Stabilization of a DC-DC "Buck" Converter: A Field Programmable Gate Array Implementation, doi: 10.1109/TIE.2011.2123857, IEEE Transactions on Industrial Electronics, 58(11), 5251-5262 (2011) 